\title{
Metadata in a LPS
}

\author{
M. PISZCZEK* \\ Institute of Optoelectronics, Military University of Technology \\ S. Kaliskiego 2, 00-908 Warsaw, Poland
}

\begin{abstract}
This paper is concerned with the use of metadata in a laser photography system. Metadata are all the data registered by additional sensors in the laser photography system. The paper is concerned with a problem of how to efficiently manage and use system metadata. The solution of using a general image metadata structure for every information unit is proposed. Various aspects of using additional data for proper image interpretation in the laser photography system are presented.
\end{abstract}

PACS: 42.30.Sy, 42.30.Tz, 02.60.-x

\section{Introduction}

Recent information systems face the necessity to manage large amounts of data. This data is often supplied by various sensors connected in systems [1-5]. Thus information systems have to face not only a management problem but also have to properly process data to extract information.

The laser photography system (LPS) being developed at the Military University of Technology has to efficiently manage data from image detectors as well as other system sensors to acquire image information successfully. It is planned to use various types of camera from the VIS-IR spectral range as well as radar. It is expected to use the system as a distributed network of information units exchanging data between themselves.

During the research project "Integrated Laser Photography System" for open space monitoring and threats detection" development of the laser photography device (LPD) as well as investigations of its abilities to combine with other sensors is realized.

The key problem is the question of how to efficiently manage and use system metadata. In the LPS a general image metadata structure for every information unit was proposed. A very important aspect of using the LPS is the possibility to use additional data for image interpretation. Proper metadata management is a condition to achieve a synergy effect for single information units as well as for multimodal sensor modules.

\section{LPD characteristics}

The LPS is a collection of sensors combining with each other for open space monitoring. The main unit of the system is a LPD.

* e-mail: mpiszczek@wat.edu.pl
Selection of system components is a result of functions necessary to realize specific tasks. Because of the scientific nature of this work it was decided to analyze the LPS in various configurations. A demonstrator of the hardware configuration is a collection of possible variants of LPS (Fig. 1).

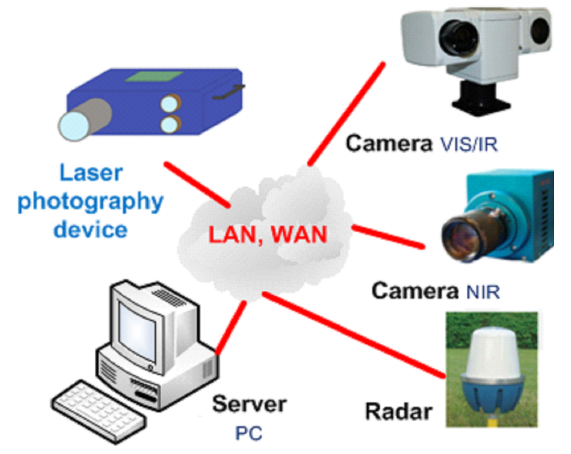

Fig. 1. Demonstrator of LPS hardware configuration.

The following units were chosen as basis elements of the LPS :

- camera: VIS/IR: FLIR PTZ-50MS,

- camera NIR: XEVA VIS-NIR,

- radar: NAVTech-W800-H.

\section{Metadata applications}

During the process of the LPS design it was decided to use individual sets of metadata for every information unit. Proposed metadata can be grouped according to applications in a single device or in a few information units. The most useful division (particularly in the range of imaging devices) of metadata is according to time, spatial, radiometric and spectral parameters [1]. The system registers data is shown in Table. From the 
various 20 available metadata there are chosen sets of additional data (except for basic data - images) registered for every information unit in a system database. In the LPD we can specify only 19 parameters (without temperature information). All other cameras register acquisition time, spatial and spectral data, tonal span and color codes. The thermovision module supplies information about work temperature range. The radar system registers all data of detected objects, localization, spatial orientation and time of data acquisition. From the point of view of the specific application the amount of data will be reduced but at the present stage of development it was decided not to reduce the amount of data.

System metadata.

TABLE

\begin{tabular}{|c|c|c|}
\hline & Type of the parameter & Format/Units \\
\hline & Temporary & \\
\hline 1 & acquisition time & YYYY-MM-DD \\
\hline 2 & lighting time & [ns] \\
\hline 3 & waiting time & [ns] \\
\hline \multirow[t]{2}{*}{4} & detection time & [ns] \\
\hline & Spatial & \\
\hline 5 & location geographic coordinate & $\overline{\text { [angle] }}$ \\
\hline 6 & orientation in space & [angle] \\
\hline 7 & angular field of lighting & [angle] \\
\hline \multirow[t]{2}{*}{8} & image resolution & [pixel] \\
\hline & Radiometry & \\
\hline 9 & laser energy & {$[\mathrm{mJ}]$} \\
\hline 10 & angular field of view & [angle] \\
\hline 11 & laser spatial profile & type \\
\hline 12 & transmission of transmitting optics & $\%$ \\
\hline 13 & transmission of receiving optics & $\%$ \\
\hline 14 & effective diameter of receiving optics & {$[\mathrm{mm}]$} \\
\hline 15 & MCP amplification & multiplier \\
\hline 16 & dynamic range & bit \\
\hline \multirow[t]{2}{*}{17} & colour encoding & type \\
\hline & Spectral & \\
\hline 18 & spectral range & {$[\mathrm{nm}]$} \\
\hline
\end{tabular}

During the project it is expected to analyze the possibility of using metadata in a basic information unit - the LPD as well as in the whole system. Usability of metadata in various configurations is defined by the system level.

\subsection{Metadata on a level of information unit}

Images are the basic type of data delivered by vision systems. This approach is sufficient for most applications [2]. There is a possibility to detect objects using images, we can also ascertain how many objects we can see in the image using basic image processing methods. Even if the image quality is inadequate we can still use traditional image processing procedures e.g. filtering. In many cases we can use images and basic image processing algorithms to extract information from an image. Unfortunately there are applications where basic methods do not give proper results. We need additional information repeatedly. In the case of conducting more complex analysis, information about context and observed scene is needed.
During measurements knowledge of data acquisition parameters (also internal orientation parameters of imaging device) is necessary [3]. It is proved that information is hidden not only in an image but also in additional data describing this image. This data can have various specifications (quantitative and qualitative) and can be categorized. The ability of extracting information from an image is a process of comparing the image with different data. Similar to human gaining abilities as he ages a technical device has to have basic knowledge and data processing algorithms implemented. As an example we can give the LPD shown in Fig. 2.

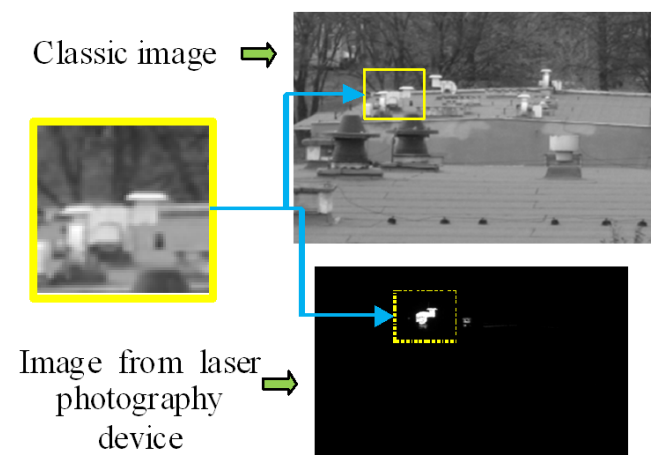

Fig. 2. Classical image and image acquired by the LPD.

If we do not know a real measurement scene and basic properties of a time-spatial framing method (the LPD works according to the time-spatial framing method), we can suppose that the image was acquired during night and shows objects "hanged in a vacuum". It can also be assumed that the image is not a raw image but a processed one or even artificially synthesized - but it is not. Despite all the issues concerning object identification we cannot extract any other information. Assuming that the object was detected we are not able to identify it based only on an image. It is only possible using metadata.

\subsubsection{Angular object size}

With knowledge of sensor geometry and imaging unit optics we can estimate the angular size of an object (Fig. 3).

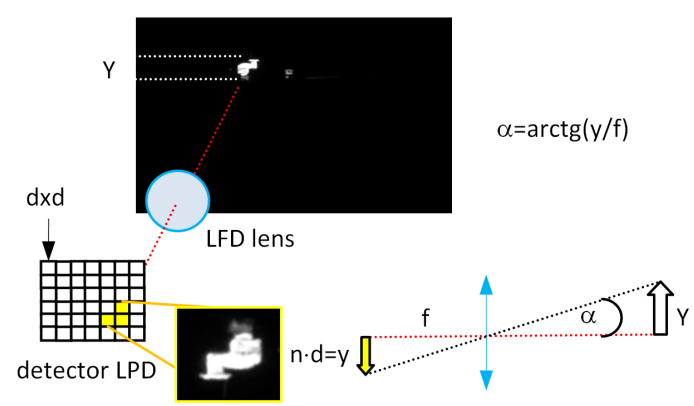

Fig. 3. Defining angular object size based on metadata. 
Information about the focal length of lens and object size is stored in a set of metadata. This data can be used to compute size using mathematical formula. Functionality of object angular dimensioning is a common property of all the imaging devices and systems. In metadata we can include additional data describing imaging unit geometric distortions by e.g. polynomial coefficients.

\subsubsection{Distance to an object}

The LPD has a unique property of estimating a distance to the observed object [5]. This functionality is a result of the methodology of the image acquisition process. The image registered in a frame is a selected fragment of space. When the depth of observation gets smaller the precision of estimating distance to an object gets bigger (Fig. 4).

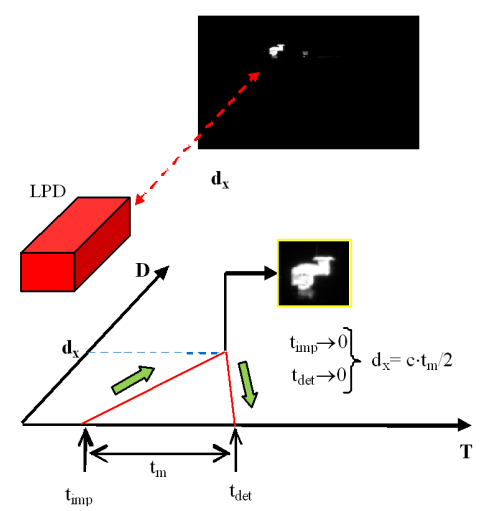

Fig. 4. Estimating the distance to an object based on metadata.

\subsubsection{Linear object size}

The ability to define linear size of an object is a derivative of distance to the object and its angular size (Fig. 5).

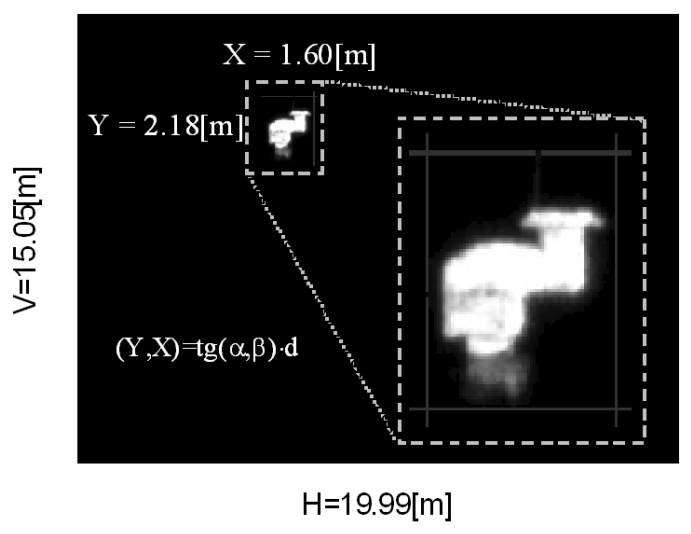

Fig. 5. Estimating linear size of an object based on metadata.

The LPD frames registered on various distances with small depth of observation allows observed scene modeling.

\subsection{Metadata on a level of information system}

During the research on the LPS demonstrator of hardware configuration was proposed. This demonstrator is a set of various configurations of information units. The LPS which is one specific version of a demonstrator has a strictly defined information potential. The information potential depends not only on the sum of single information units but also on unique capabilities of a combination of units. To achieve a synergy effect knowledge of metadata is necessary. Some of the LPD properties described above are unique e.g. spatial selectivity of observation. Using this functionality in specific tasks can be difficult especially during open space monitoring. A selective search of a space can be very time-consuming. Is there any solution that can make monitoring more efficient? We should start by looking for a solution in a field not connected with imaging techniques - a cognitive psychology. A human would be overloaded with huge amounts of data coming from various senses if did not keep attention. Attention is a kind of module filtering excess information. Thanks to sight perception moving objects can attract our attention and acoustic signals can focus our attention on a source of sound etc.

\subsubsection{LPD cooperation with other vision systems}

Classic imaging devices working in spectral range e.g. VIS-IR can register a space limited by an angular field of view. There is no such limitation in distance to observed object. Both VIS and IR cameras acquire information about direction to observed object as well as its angular size. Object detection by a VIS/IR camera can be a focusing factor for a system [4]. Application capabilities of such information can be strictly connected with metadata of every single information unit. Even if all cameras are spatially separated information about localization, spatial orientation and object observation direction can allow computation of observation coordinates for the LPD (Fig. 6).

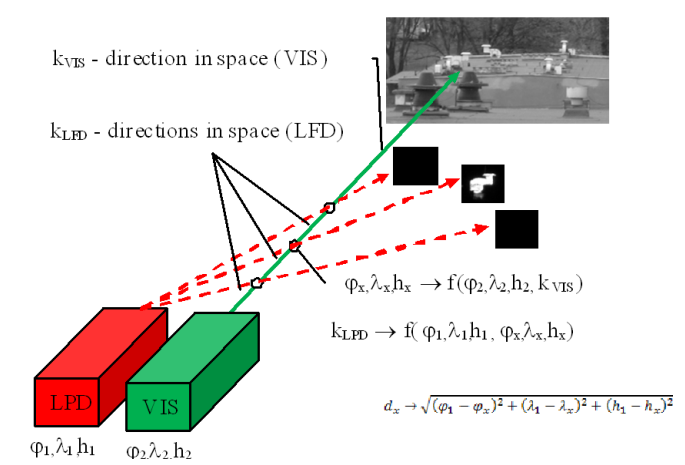

Fig. 6. Use of metadata from the LPS vision devices.

Information from VIS/IR cameras can be supplemented by data from the LPD. Images from the LPD can be more suitable for object identification. Initially a detected object can be localized in a 3D space and even its geometry in linear coordinates can be measured. 


\subsubsection{The $L P D$ in combination with radar}

One of properties of a radar is an ability to provide $3 \mathrm{D}$ coordinates of an object. This means that sending information to a system can be a focusing factor for an information system. The role of the radar is to detect objects and provide information about direction and speed of move.

Metadata connected with the radar device, its location, spatial localization and coordinates of the object detected are input parameters for the LPS (Fig. 7).

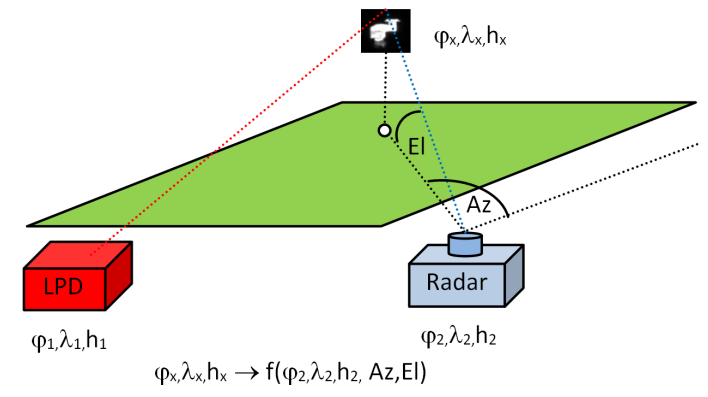

Fig. 7. Utilization of radar metadata in the LPS.

Fuller information about the object can be delivered by LPD which using localization data can acquire images for object identification or geometry description.

\section{Summary}

Recent information systems frequently manage large amounts of data. In imaging systems image data is not the only important information. Very important data can be stored in image describing data - image metadata. During the development process of a new information system the potential benefits of using basic data should be included.
Knowledge and the ability to use this apparently secondary data can have a key sense for identification of information system potential. According to examples shown in this article metadata are equally important in the case of a single information unit (LPD) as well as all the relations between various information units (LPS: LPD-radar). Knowledge of metadata describing the process of registration of time-spatial frame is necessary for acquisition and interpretation of images registered by the LPD. For geometry analysis of observed scene information about the internal orientation of the imaging unit is needed. During the analysis and data fusion process, using data from various information units and external orientation parameters is useful. Possible image fusion does not have to be achieved using only imaging material because during image processing metadata can also be used.

\section{Acknowledgments}

This work was funded by the Polish Ministry of Science and Higher Education (project OR00000312).

\section{References}

[1] R. Mathis, L. Caughey, in: Proc. HICSS '05, Ed. Y. Wang, University of Hawai, Vol. 1, 2, 2005.

[2] X. Hua Wang, S. Wang, Technologies for Ambient Intelligences, University of Genoa, Italy 2005.

[3] S. Zillner, Proceedings of the 2011 IEEE International Conference on Information Reuse and Integration, IEEE SMC, Piscataway 2011, p. 412.

[4] M. Piszczek, Acta Phys. Pol. A 120, 716 (2011).

[5] M. Piszczek, K. Rutyna, M. Szustakowski, Europ. Phys. J. Spec. Top. 154, 153 (2008). 